Correl at $i$ on between the resul ts of charge deep- I evel transi ent spect roscopy and ESR techni ques for undoped hydrogenat ed anor phous silicon

\begin{tabular}{|c|c|}
\hline 著者 & $\begin{array}{l}\text { Na?daz?dy V., Durn? R., Thur zo I., Pi nc?i ?k } \\
\text { E., Ni shi da A., Shi mi zu J., Kumeda M nor u, } \\
\text { Shi mi zu Tat suo }\end{array}$ \\
\hline $\begin{array}{l}\mathrm{j} \text { our nal or } \\
\text { publ i cat } \mathrm{i} \text { on } \mathrm{titl} \text { e }\end{array}$ & $\begin{array}{l}\text { Physi cal Revi ew B - Condensed Natter and } \\
\text { Nat er i al s Physi cs }\end{array}$ \\
\hline vol une & 66 \\
\hline number & 19 \\
\hline page $r$ ange & 195211- 1- 195211- 8 \\
\hline year & 2002-11- 01 \\
\hline URL & ht t p: //hdl . handl e. net /2297/3508 \\
\hline
\end{tabular}




\title{
Correlation between the results of charge deep-level transient spectroscopy and ESR techniques for undoped hydrogenated amorphous silicon
}

\author{
V. Nádaždy, ${ }^{1}$ R. Durný,${ }^{2}$ I. Thurzo, ${ }^{1}$ E. Pinčík,${ }^{1}$ A. Nishida, ${ }^{3}$ J. Shimizu, ${ }^{4}$ M. Kumeda,${ }^{4}$ and T. Shimizu ${ }^{4}$ \\ ${ }^{1}$ Institute of Physics SAS, Dúbravská cesta 9, 84228 Bratislava, Slovak Republic \\ ${ }^{2}$ Department of Physics, FEI STU, Ilkovičova 3, 81219 Bratislava, Slovak Republic \\ ${ }^{3}$ Department of Applied Physics, Faculty of Science, Fukuoka University, Fukuoka 814-0180, Japan \\ ${ }^{4}$ Faculty of Engineering, Kanazawa University, Kanazawa 920-8667, Japan
}

(Received 9 January 2002; published 18 November 2002)

\begin{abstract}
Results of charge deep-level transient spectroscopy (DLTS) and electron spin resonance (ESR) measurements on undoped hydrogenated amorphous silicon $(a-\mathrm{Si}: \mathrm{H})$ clearly demonstrate that a group of gap states with a mean energy of $0.82 \mathrm{eV}$ as observed in charge DLTS experiments for $a$-Si:H based metal/oxide/ semiconductor structure is the same as the $g=2.0055$ ESR defect (the $D_{z}$ component). This correlation provides a distinct marker for charge DLTS technique. We obtained a very good fit to spectra obtained on undoped $a-\mathrm{Si}: \mathrm{H}$ in the annealed state whilst there is some discrepancy between the experimental and simulated spectra for the light-soaked state. The first quantitative comparison of defect pool model with gap states directly observed by charge DLTS offers not only additional data for more accurate identification of all the intrinsic and light-induced defects. This also renders distinct counter-evidence to recently published conjectures about the creation of another charged defect during early stage of Staebler-Wronski effect. By contrast, our presented results clearly argue for opposite process, i.e., decay of positively charged defect states $D_{h}$.
\end{abstract}

DOI: 10.1103/PhysRevB.66.195211

PACS number(s): 71.23.Cq, 71.55.Jv

\section{INTRODUCTION}

The knowledge of gap states in hydrogenated amorphous silicon $(a-\mathrm{Si}: \mathrm{H})$ is of fundamental importance. It is not only the key to understanding the basic physics of the material but it controls the performance of all device applications. In $a$-Si:H, gap states can be divided into band-tail states and deep defect states. Deep levels associated with defect states strongly influence the charge carrier transport and lifetime.

Electron spin resonance (ESR) is one of the few experimental techniques which can provide information on the microscopic structure of defects in semiconductors (including the amorphous ones) and the ESR results are considered unambiguous. For example, results of ESR measurements of neutral dangling bond occupied by one electron with $g$ $=2.0055$ are regarded as experimental standard. However, ESR sensitivity is quite low in case of thin films with a low spin density, and information is obtained only about paramagnetic defects, i.e., defects with an unpaired electron. In contrast, the deep level transient spectroscopy (DLTS) is extremely sensitive and it can detect not only deep levels of diamagnetic defects. It is routinely used to determine energy levels and defect concentrations in semiconductors. Actually, DLTS is perhaps the most common technique for measuring deep levels in crystalline semiconductors. Lang et al. ${ }^{1}$ and Cohen and Lang ${ }^{2}$ showed that DLTS is a promising method for monitoring the electronic density of states $g(E)$ also for $a$-Si:H. Their analysis and experiments on $n$-type $a$-Si:H demonstrated the sensitivity of DLTS to changes in $g(E)$. DLTS data are very close to the shape of $g(E)$ and were used as the first trial function for iterative fitting procedure of DLTS spectra in order to obtain the bulk density of states for $a$-Si:H. This group also reported ${ }^{3}$ on the identification of the midgap defect band in $n$-type $a$-Si:H observed by DLTS with the ESR signal with $g=2.0053$. In another paper ${ }^{4}$ they present measurements of thermally induced reversible changes in the capacitance of Schottky barrier following a 10 min bias annealing at $473 \mathrm{~K}$. These changes were due to an increase of the shallow donor concentration in the bulk approximately an order of magnitude. Nevertheless, it was not possible to decide whether the doping effect is connected with an activation of the electrically inactive donors or is inherent to $a$-Si:H network itself. A common feature of the above mentioned experiments is that DLTS technique was applied to doped $a-\mathrm{Si}: \mathrm{H}$. Since it was proved ${ }^{5}$ that metastability of electrical properties due to changes in the gap states distribution is inherent to the network only consisted of $\mathrm{Si}$ and $\mathrm{H}$ atoms, the study of gap states in undoped $a$-Si:H has a key importance.

The original capacitance DLTS failed for undoped $a$-Si:H and other high resistivity semiconductors, because a low measurement frequency is needed to make the dielectric response turn-on temperature low enough to observe the gap states over an appreciable fraction of the gap. Therefore, we have used the charge version of the DLTS which is able to resolve $g(E)$ in undoped $a$-Si:H. ${ }^{6}$ The charge DLTS profits from the direct detection of thermally emitted carriers in the form of current in the external circuit. As a result of the first DLTS measurements on undoped $a$-Si:H based MOS structure, we have observed three groups of states, with mean energies of $0.63,0.82$, and $1.25 \mathrm{eV}$, in a good agreement with the existence of the $D_{h}, D_{z}$, and $D_{e}$ dangling bond states, respectively, predicted by improved defect-pool model. ${ }^{7-10}$ All of these referred papers are based on the defect-pool concept which was excellently described in a classic paper by Winer. ${ }^{11}$ According to the defect-pool model, the defect chemical potential depends on the Fermi energy and a shift of the Fermi level at the equilibration temperature should 
change the proportions among the densities of $D_{h}, D_{z}$, and $D_{e}$ components. In fact, our charge DLTS measurements followed a suggestion of Winer. ${ }^{11}$ The idea of this experiment is to shift the Fermi level of an equilibrated $a-\mathrm{Si}: \mathrm{H}$ film in a metal/insulator/semiconductor (MIS) structure by applying a bias voltage, and to monitor the resulting change in the defect distribution as the film re-equilibrates. In the light of this finding, the observation of a reversible field-induced doping effect in $a-\mathrm{Si}: \mathrm{H}^{4}$ can be also viable explained.

To utilize the potential of the DLTS technique, it is necessary to set unambiguous relations to other well established methods in this field. Concerning the ESR technique, it is obviously of interest to prove that the gap states which represent a group of $D_{z}$ defects with mean energy of $0.82 \mathrm{eV}$, as observed in charge DLTS experiments on undoped $a-\mathrm{Si}: \mathrm{H}$ based MOS structure, is the same as the $g=2.0055$ ESR defect (the $D_{z}$ component). Our preliminary results ${ }^{12}$ indicated that it was indeed the case. In this paper, we present the results of charge DLTS and ESR measurements on annealed and extensively light-soaked samples of undoped $a$-Si:H. The light-soaking time dependence of the amplitude of the charge DLTS and ESR signals is discussed in terms of the shift of the Fermi level and photoinduced increase in network disorder of $a$-Si:H observed by polarized electroabsorbtion (PEA). ${ }^{13,14}$ All things considered there is a strong evidence that the results of charge DLTS and ESR measurements on $D_{z}$ component correlate well and the DLTS peak with mean activation energy of $0.82 \mathrm{eV}$ can be considered as a marker in relation to ESR technique. We compare the experimental charge DLTS spectra with those obtained as a result of simulations based on the improved defect-pool model. ${ }^{7,9}$ Also, the electronic density of states, $g(E)$, is reconstructed on the basis of charge DLTS results obtained on undoped $a$-Si:H samples in annealed and light-soaked states. Reconstructed $g(e)$ clearly shows decay of positively charged defect states $D_{h}$ during early stage of StaeblerWronski effect (SWE) and disprove the creation of new charged defect states assumed in recent works. ${ }^{15-18}$

\section{EXPERIMENT}

Undoped $a$-Si:H layers of the thickness of $1 \mu \mathrm{m}$ were deposited on $n^{+}$-type crystalline Si substrates in $13.52 \mathrm{MHz}$ rf-excited parallel plate plasma enhanced chemical vapor deposition system from pure $\mathrm{SiH}_{4}$ at the plasma power density of $0.3 \mathrm{~W} / \mathrm{cm}^{2}$ and the nominal substrate temperature of $350^{\circ} \mathrm{C}$ (the actual temperature is expected to be $250-300^{\circ} \mathrm{C}$ ). For successful charge DLTS experiments on undoped a-Si:H a very thin insulating layer (VTIL) has to be created in the surface region of $a$-Si:H (Ref. 19) in order to reduce the leakage currents of the semiconductor structure under investigation to negligible values with respect to charge transients. The VTIL of approximately $5 \mathrm{~nm}$ thickness is formed by Ar beam bombardment (ArBB) on the $a-\mathrm{Si}: \mathrm{H}$ film in vacuum by $300 \mathrm{eV}$ argon atoms with the fluence of $10^{16} \mathrm{~cm}^{-2}$ for $5 \mathrm{~min}$. After ArBB, the vacuum system was filled with molecular oxygen. The temperature of the sample during the $\mathrm{ArBB}$ does not exceed $50^{\circ} \mathrm{C}$ as measured at the back side of the sample. The annealing of the samples after

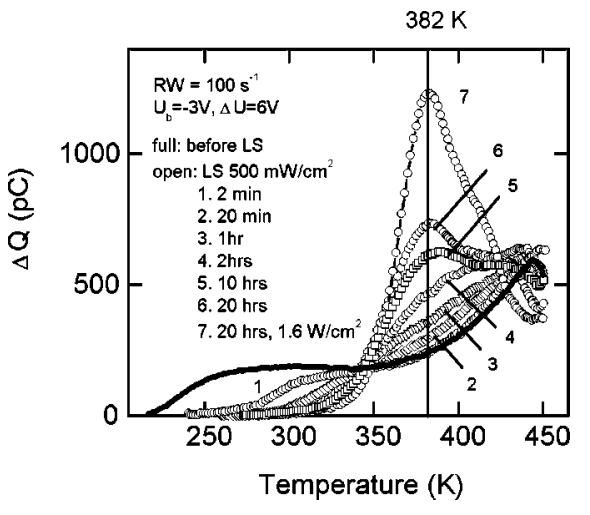

FIG. 1. Charge DLTS spectra of undoped $a-\mathrm{Si}: \mathrm{H}$ in annealed state (full line) and after light soaking (open symbols). Measurements were performed with applied bias $U_{b}=-3 \mathrm{~V}$, excitation pulses $\Delta U=6 \mathrm{~V}$, and rate window $R W=100 \mathrm{~s}^{-1}$.

both deposition and $\mathrm{ArBB}$ was done at $200^{\circ} \mathrm{C}$ for $5 \mathrm{~h}$. As we have shown, the VTIL has an almost stochiometric composition of $\mathrm{SiO}_{x}(x \sim 2.0){ }^{19}$ Thus, after evaporation of top semitransparent Al contacts, a MOS structure is obtained. The area of the contacts was $3.4 \times 10^{-3} \mathrm{~cm}^{2}$ with a transparency of $40 \%$ in the visible-light region. For ESR measurements $a$-Si:H films were deposited on silica glass substrates and were exposed to the same treatments as those for charge DLTS experiments. The light soaking of $a$-Si:H samples was performed at room temperature with infrared-filtered white light of two different intensities of 1.25 and $4 \mathrm{~W} / \mathrm{cm}^{2}$. Considering the $40 \%$ transparency of the $\mathrm{Al}$ contacts it means effective light intensities of $500 \mathrm{~mW} / \mathrm{cm}^{2}$ and $1.6 \mathrm{~W} / \mathrm{cm}^{2}$. During the light soaking, the samples were placed on a cooler to avoid any increase of the temperature. Charge DLTS experiments were performed using a homemade advanced transient charge processor. All charge DLTS spectra were obtained with the following parameters: applied bias $U_{b}=-3 \mathrm{~V}$, excitation pulses $\Delta U=6 \mathrm{~V}$, and the rate window $R W=100 \mathrm{~s}^{-1}$. In addition, feedback charge capacitance $^{20}$ vs voltage $(C-V)$ curves were measured at different temperatures with sampling time of $10 \mathrm{~ms}$ which corresponds to the inverse of $R W=100 \mathrm{~s}^{-1}$. ESR measurements were performed with a JEOL X-band spectrometer at room temperature.

\section{RESULTS}

Results of charge DLTS measurements on annealed and light-soaked $a$-Si:H samples are shown in Fig. 1. The annealed spectra are characterized by a broad signal without any distinct peak. Successive LS causes removal of lowtemperature part of the spectra. After short delay, an increase of the signal around $400 \mathrm{~K}$ is observed. For prolonged LS, this signal develops in a distinct peak around $382 \mathrm{~K}$ and simultaneously the high-temperature part of the signal slightly decreases. The activation energy and exponential prefactor $e_{0}$ of the peak at $382 \mathrm{~K}$ determined by the standard Arrhenius' plot is $0.82 \mathrm{eV}$ and $6 \times 10^{12} \mathrm{~s}$, respectively. The energy of the peak and its successive increase with prolonged LS indicates a well established experimental fact - 


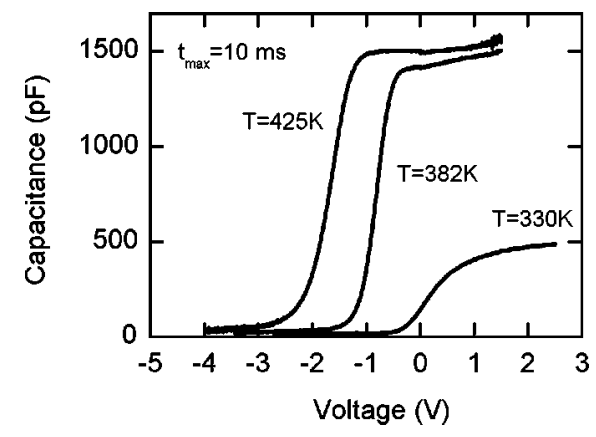

FIG. 2. The feedback-charge capacitance vs voltage $(C-V)$ curves for three different temperatures of $330 \mathrm{~K}, 382 \mathrm{~K}$, and $425 \mathrm{~K}$. Setting bias voltage of $-3 \mathrm{~V}$ and excitation pulses of $6 \mathrm{~V}$, the depletion region represents the whole $a$-Si:H film beneath the top electrode and contribute to the charge DLTS signal.

the creation of new, so-called metastable defects. We assume that observed charge DLTS spectra are due to a continuum of thermal emission rates determined by an energy distribution of noninteracting defect states. The rate window concept of DLTS technique extended to this case means that contribution to the DLTS signal for particular deep state at energy $E$ appears at temperature determined by the electron thermalemission rate

$$
e_{n}=e_{0} \exp \left(-\frac{E}{k T}\right)
$$

where $E$ is the energy position of gap states with respect to the conduction band edge. Thus the DLTS rate window also serves as an energy filter. The exponential prefactor $e_{0}$ is given by the product of the electron capture cross section, $\sigma_{n}$, the average thermal velocity of electrons $\left\langle v_{n}\right\rangle$, and the effective density of states of the conduction band $N_{c 0}$. The energy resolution for continuously distributed gap states we already discussed in our previous paper. ${ }^{21}$ It was shown that double boxcar processing of the transient response with sampling times $t_{1}$ and $2 t_{1}$ gives the energy resolution of $E / 9.3$. Therefore, the charge DLTS signal at $382 \mathrm{~K}$ includes charge from states with energy of $0.82 \pm 0.05 \mathrm{eV}$. This estimate is lower than the correlation energy of the dangling bond states. Thus, the height of DLTS signal at $382 \mathrm{~K}$ is directly proportional to the density of states occupied by single electron.

The ESR signal comes from the whole $a$-Si:H film. To compare this signal with the amplitude of the charge DLTS spectra, it is desirable to collect the charge DLTS signal from the full width of $a$-Si:H film beneath the top contact. Let us inspect Fig. 2, where $C-V$ curves are shown for three different temperatures of $330 \mathrm{~K}, 382 \mathrm{~K}$, and $425 \mathrm{~K}$, at which the $D_{h}, D_{z}$, and $D_{e}$ components mainly contribute to the charge DLTS signal. The sampling time of $10 \mathrm{~ms}$ means that the excess capacitance with respect to the capacitance of $a-\mathrm{Si}: \mathrm{H}$ film $C_{a-\mathrm{Si}: \mathrm{H}}=36 \mathrm{pF}$, is only due to charges emitted with a thermal rates not lower than the inverse of $10 \mathrm{~ms}$. The capacitance of $36 \mathrm{pF}$ indicates that crossovers of the Fermi level with deep states, which emit charges contributing to the capacitance, are pushed into the bottom $a-\mathrm{Si}: \mathrm{H} / \mathrm{n}^{+} c$-Si interface. On the other hand, the accumulation capacitance (the

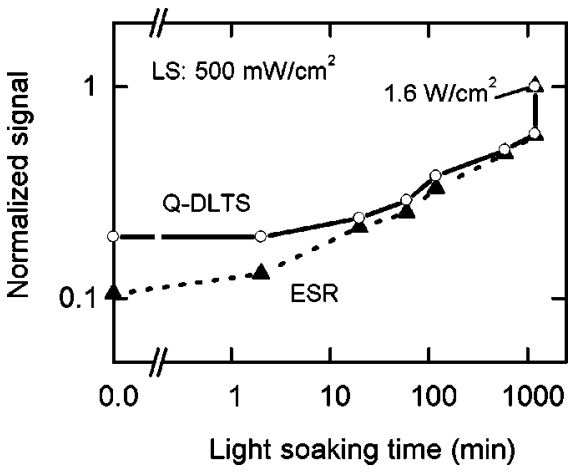

FIG. 3. Light soaking time dependence of the charge DLTS and ESR signal intensities obtained on undoped $a$-Si:H. Signals intensities recorded after the final $20 \mathrm{~h}$ of light illumination with $1.6 \mathrm{~W} / \mathrm{cm}^{2}$ light intensity were made identical and normalized to " 1. ."

capacitance of the oxide layer $C_{o x} \approx 1500 \mathrm{pF}$ ), is obtained when the crossovers of the Fermi level with the gap states move towards the top $a$-Si:H/SiO 2 interface. Full accumulation capacitance is not reached at the lowest temperature of $330 \mathrm{~K}$. We explain this fact by the higher density of $D_{z}$ states in the subsurface region of the undoped $a-\mathrm{Si}: \mathrm{H}^{22}$ Namely, the emission rate from $D_{z}$ states is too low with respect to the sampling time of $10 \mathrm{~ms}$ and therefore this subsurface region contributes to the width of the insulating layer of the MOS structure under investigation. These $C$ - $V$ curves justify our assumption that at the bias voltage and excitation pulses of -3 and $6 \mathrm{~V}$, respectively, the depletion region practically corresponds to the width of the $a-\mathrm{Si}: \mathrm{H}$ film. Therefore, the amplitude of the charge DLTS spectra at the temperature of $382 \mathrm{~K}$ can be taken as a measure of the total density of the neutral $D_{z}$ component in the $a$-Si:H film.

ESR measurements were also performed on annealed and LS samples of undoped $a-\mathrm{Si}: \mathrm{H}$. To correlate the time evolution of the amplitude of the charge DLTS signal at the temperature of $382 \mathrm{~K}$ and ESR signals due to LS, we plot both of them as a function of time in Fig. 3. Charge DLTS and ESR signals of the $D_{z}$ component recorded after the final light illumination $\left(20 \mathrm{~h}, 1.6 \mathrm{~W} / \mathrm{cm}^{2}\right)$ were made identical and normalized to " 1 ." The reason for doing so is that the downward shift of the Fermi level has already been finalized. In addition, at the end of light soaking both signals have a very good signal to noise ratio while in the annealed state just the opposite holds for ESR signal intensity. Although its error bars are approximately $50 \%$ of the measured experimental value, we are confident about its tendency to increase with light soaking.

\section{DISCUSSION OF EXPERIMENTAL RESULTS}

As a result of light soaking quick and precipitous decrease of dark and photoconductivities is observed in $a-\mathrm{Si}: \mathrm{H}$ samples. This experimental fact can be explained by a downward shift of the Fermi level towards midgap due to the removal of $D_{h}$ defects, which is manifested with decay of low-temperature part of charge DLTS spectra (see Fig. 1). The shift of the Fermi level into the ascending shoulder of 
traps near midgap also causes an increase of the ESR signal in the initial stage of light soaking (see Fig. 3). It should be noted, however, that the ESR measured increased density of $D_{z}$ defects is not due to the creation of new, so-called metastable $D_{z}$ defects. It reflects on the Fermi level position in a region of increased density of already existing, so-called native, $D_{z}$ defects. A similar effect was observed on $n$-type $a$-Si:H diode junction where the changes in the dark absorption ESR signal was achieved by modulating the width of the depletion region of $n$-type $a$-Si:H layer. ${ }^{3}$ In contrary to the ESR technique, the charge DLTS detected $D_{z}$ defect density remains flat during the first several minutes of intense $\left(500 \mathrm{~mW} / \mathrm{cm}^{2}\right)$ white light illumination (see Fig. 3). The reason for this is that our charge DLTS measurements were performed with large excitation pulse $(\Delta U=6 \mathrm{~V})$ and so for a given temperature and rate window we measure practically all $D_{z}$ defects. Therefore, a shift of the Fermi level does not affect the measured charge DLTS signal. This important detail of degradation process, not creation of metastable $D_{z}$ defects, is consistent with changes in subgap absorption measured with dual beam photoconductivity by Jiao et al. ${ }^{23}$ The authors reported on no discernible difference between the annealed state and after the half-minute with air mass 1 illumination. Only after that time, the subgap absorption starts to increase which is due to increased density of $D_{z}$ defects.

Despite some differences between the results of ESR and charge DLTS measurements in the early stage of light soaking the results demonstrate that the deep level which represent a group of gap states with mean energy of $0.82 \mathrm{eV}$, as observed in charge DLTS experiments on undoped $a-\mathrm{Si}: \mathrm{H}$ based MOS structure, is the same as the $g=2.0055$ ESR defect (the $D_{z}$ component). Supporting evidence for the existence of some kind of precursor to the $D_{z}$ defect is coming from PEA measurements. ${ }^{13,14}$ They indicate a strong increase of structural disorder upon light soaking which saturates within a few minutes. It correlates well with our observation mentioned above that before creation of the metastable $D_{z}$ defects, $D_{h}$ defects are removed in this early stage of light soaking. Thus, photoinduced structural changes occur during the initial stage of light soaking even before the creation of metastable dangling bond defects. In the later stage of lightsoaking creation of metastable dangling bond defects takes place as well. ${ }^{24}$ The following, second step of the degradation process is connected with the creation of new, metastable, $D_{z}$ defects with the known $t^{1 / 3}$ time dependence. Also, our small-signal charge transient measurements ${ }^{25}$ as a function of the bias voltage $U_{b}$, which provide information about the spatial distribution of all $\left(D_{h}, D_{z}\right.$, and $\left.D_{e}\right)$ components, clearly demonstrate that before the creation of metastable $D_{z}$ defects the $D_{h}$ defects are completely removed by light soaking. Valuable experimental results on initial rapid lightinduced changes of photoconductivity and electron mobilitylifitime product were recently published by two groups. ${ }^{15-18}$ They also reported significant changes in degradation kinetics. However, their explanation of observed changes is completely opposite. They assume creation of positively charged defects in the course of early stage of degradation. Since they assume existence of another defects indirectly from measurements of optical absorption and electron mobility-lifetime product, their interpretation is less probable. Our opposite assertion is based on direct observation of decay of $D_{h}$ states. They take the role of donors and their decay causes the well-known drop in dark and photoconductivities accompanied with changes of degradation kinetics and electron mobility-lifetime product. Preliminary simulation performed with amorphous semiconductor analysis (ASA) software package $^{26}$ also confirms a consistency of this explanation. It is worth to note that similar effect of doping was already observed in bias annealing experiments by capacitance measurements ${ }^{4}$ as well as by charge measurements ${ }^{6}$ and can be understood in the frame of defect-pool model.

\section{SIMULATION OF THE CHARGE DLTS SPECTRA USING GAP-STATE DISTRIBUTION FROM DEFECT-POOL MODEL}

Experimental results give reason for further analysis of the charge DLTS spectra in order to obtain more detailed information about the energy distribution of the gap states. It is of interest to compare the experimental charge DLTS spectra obtained on annealed and light soaked samples of undoped $a$-Si:H with the theoretical ones obtained as the best fit to experimental data points by simulation. A comprehensive study by Cohen and Lang $^{2}$ is devoted to numerical analysis of thermal transient and ac dynamic response of a Schottky-barrier space charge region for a semiconductor with an arbitrary continuous density of the gap states $g(E)$. Their analysis shows that the raw DLTS data is nearly proportional to $g(E)$. They used this fact for self-consistent iteration of DLTS spectra where as a starting trial function of $g(E)$ was directly taken these experimental DLTS spectra. Our calculations of charge DLTS spectra are performed using a modified formula of Cohen and Lang ${ }^{2}$

$$
\Delta Q=A q \frac{\Delta w}{2} \int_{E_{1}}^{E_{2}} g(E)\left[\exp -\left(\frac{t_{1}}{e_{n}}\right)-\exp -\left(\frac{2 t_{1}}{e_{n}}\right)\right] d E .
$$

This formula describes the emission from a distribution of noninteracting defect states and is valid only if the trap emission occurs over the entire depletion width $\Delta w$. Here, we ignore spatial changes of $g(E)$. It means the gap states distribution $g(E)$ obtained from the fitting procedure represents an averaged value for the whole region below the top electrode. Also, the factor $C_{\mathrm{ox}} /\left(C_{\mathrm{ox}}+C_{a-\mathrm{Si}: \mathrm{H}}\right)$ for the $M 0 S$ structure is omitted because the capacitance of the oxide layer $\left(C_{\mathrm{ox}} \approx 1500 \mathrm{pF}\right)$ is much higher than the capacitance of the $a$-Si:H film $\left(C_{a-\mathrm{Si}: \mathrm{H}}=36 \mathrm{pF}\right)$. Product under the integral represents convolution of $g(E)$ with the correlation function of the double boxcar processing ${ }^{27}$ of the transient response. The electron thermal emission rate $e_{n}$ for a particular gap states at energy $E$ is given by the relation (1). Our previous charge DLTS measurements ${ }^{6}$ showed that the exponential prefactor $e_{0}$ was a function of energy and could be expressed using the Mayer-Neldel (MN) rule ${ }^{28}$

$$
e_{0}=e_{00} \exp \left(\frac{E}{E_{0}}\right)
$$


where $E_{0}$ is the $\mathrm{MN}$ energy and $e_{00}$ is an energy independent constant. These parameters are determined from the previous results for the activation energies and the exponential prefactors for the charge DLTS peaks corresponding to $D_{h}, D_{z}$, and $D_{e} \cdot{ }^{6}$ The abovementioned measurements also demonstrated that evolution of DLTS spectra after the preequilibrium preparation by bias annealing could be qualitatively described by the improved defect-pool model. ${ }^{7-10}$ Therefore, we used this model for the simulation of charge DLTS spectra and employed two formulas for description of the density of dangling bond states $D(E)$. The first one is given by Powell and Deane ${ }^{7}$

$$
D(E)=\gamma_{P D}\left[\frac{2}{f^{0}(E)}\right]^{\rho k T / E_{v 0}} P\left(E+\frac{\rho \sigma^{2}}{E_{v 0}}\right),
$$

where

$$
\begin{gathered}
\gamma_{P D}=\left[\frac{N_{v 0} 2 E_{v 0}^{2}}{2 E_{v 0}-k T}\right]^{\rho} N_{H}^{1-\rho} \exp \left[-\frac{\rho}{E_{v 0}}\left(E_{p}-E_{v}-\frac{\rho \sigma^{2}}{2 E_{v 0}}\right)\right], \\
f^{0}(E)=\frac{2 \exp \left[\frac{\left(E_{F}-E\right)}{k T}\right]}{1+2 \exp \left[\frac{\left(E_{F}-E\right)}{k T}\right]+\exp \left[\frac{\left(2 E_{F}-2 E-U\right)}{k T}\right]}, \\
P(E)=\frac{1}{\sigma \sqrt{2 \pi}} \exp \left[-\frac{\left(E-E_{p}\right)^{2}}{2 \sigma^{2}}\right],
\end{gathered}
$$

and

$$
\rho=\frac{E_{v 0}}{E_{v 0}+k T} .
$$

The second formula was derived by Schumm ${ }^{9}$

$$
D(E)=\gamma_{S}\left[\frac{2}{f^{0}(E)}\right]^{k T / 2 E_{v 0}} P\left(E+\frac{\sigma^{2}}{2 E_{v 0}}\right),
$$

where

$$
\begin{gathered}
\gamma_{S}=\left(2 N_{v 0} \alpha \beta\right)^{\rho^{\prime}}\left(2 N_{H}\right)^{1-\rho^{\prime}} \delta, \\
\alpha=\frac{\pi k T}{2 \sin \pi k T / 2 E_{v 0}}, \\
\beta=\int P(E)\left[\frac{2}{f^{0}} \exp -\frac{E}{k T}\right]^{\left(k T / 2 E_{v 0}\right)\left(1-k T / E_{v 0}\right)} d E, \\
\delta=\exp \left[\frac{\sigma^{2}}{2\left(2 E_{v 0}\right)^{2}}-\frac{E_{p}-E_{v}}{2 E_{v 0}}\right],
\end{gathered}
$$

and

$$
\rho^{\prime}=\left(1+k T / E_{v 0}\right)^{-1} .
$$

These formulas represent the density of dangling bond states at equilibrium, which is maintained for temperatures above the equilibration temperature $T^{*}=500 \mathrm{~K}$. We have to use temperature $T^{*}$ for calculation of $D(E)$ even at temperatures below $T^{*}$ because equilibration time is too long and $D(E)$ is assumed to be frozen in. Differences between the formulas arise from different treatments of the equilibrium reactions between week and dangling bonds. Nevertheless, both solutions result in qualitatively similar energy distribution of the gap states with a small difference in the total density of gap states. The absolute density is given by the scaling factors $\gamma_{P D}$ (5) and $\gamma_{S}$ (10). Relations (6) and (7) represent the occupancy function of the gap states occupied by single electron and the defect-pool function $P(E)$, which is assumed to have a Gaussian distribution, respectively. The following parameters enter into the relations (4)-(14): the valence-band tail slope $E_{v 0}$, the density of states at the valence band edge $N_{v 0}$, the hydrogen concentration $N_{H}$, the defect-pool width $\sigma$, the most probable energy in the distribution of available sites for defect formation $E_{p}$, the Fermi level position with respect to the valence band $E_{F}$, and the Boltzman constant $k$. The density of states in the conduction-band tail and the valence-band tail has the following forms:

$$
\left.N_{c b}=N_{c 0} \exp \left(E-E_{c}\right) / E_{c 0}\right)
$$

and

$$
N_{v b}=N_{v 0} \exp \left(-E / E_{v 0}\right),
$$

respectively. The total one-electron density of states was taken as a sum of gap states due to dangling bonds ${ }^{7}$ and the conduction- and valence-band tails

$$
\begin{aligned}
g(E)= & D[E+k T \ln (2)]+D[E-U-k T \ln (2)] \\
& +N_{c b}(E)+N_{v b}(E) .
\end{aligned}
$$

Using the above mentioned expressions for describing the gap states, we simulated charge DLTS spectrum of the sample in the annealed state (see full line in Fig. 1). Four parameters of the fitting procedure: position of the defect pool $E_{p}$, the width of the defect pool $\sigma$, correlation energy $U$, and the integral prefactor $\Delta Q_{0}$, of the formula (2) were allowed to change. Other parameters entering in the relations (4)-(14) were taken from experimental results. The position of the Fermi level was determined as the activation energy of the dark conductivity. Remaining parameters were adopted from literature for a standard quality a-Si:H film. Both parameters fixed and fitted are summarized in Tables I and II, respectively. Integration boundaries $E_{1}$ and $E_{2}$ were first chosen to fit part of DLTS spectra between $270 \mathrm{~K}(1.40 \mathrm{eV})$ and $440 \mathrm{~K}(0.60 \mathrm{eV})$. Then, energy boundaries $E_{1}$ and $E_{2}$ were successively moved towards valence and conduction bands, respectively, until we obtained the best fit of the experimental spectrum. It was achieved for $E_{1}=0.47 \mathrm{eV}$ and $E_{2}=1.46 \mathrm{eV}$.

Schumm ${ }^{9}$ treated also the case of nonequilibrium distribution of the gap states achieved after LS. He derived the expression for the metastable steady-state density of states

$$
D_{S S}(E)=\gamma_{S}^{*}\left[\frac{2}{f^{0 *}(E)}\right]^{k T / 2 E_{v 0}} P\left(E+\frac{\sigma^{2}}{2 E_{v 0}}\right)\left[\frac{n p}{n_{0} p_{0}}\right]^{\rho, k T / 2 E_{v 0}} \text {. }
$$


TABLE I. Summary of the defect pool parameters taken as fixed for simulation of the charge DLTS spectra in Fig. 4 by models of Powell and Dean (Ref. 7) and Schumm (Ref. 9). We used the values typical for standard quality of $a-\mathrm{Si}: \mathrm{H}$ film.

\begin{tabular}{lcc}
\hline \hline Fixed parameter & Symbol & Value \\
\hline Band gap & $E_{g}$ & $1.8 \mathrm{eV}$ \\
Fermi level with respect & $E_{F}$ & $1.1 \mathrm{eV}$ \\
$\quad$ to VB edge & $T^{*}$ & $500 \mathrm{~K}$ \\
Freeze-in temperature & $N_{H}$ & $5 \times 10^{21} \mathrm{~cm}^{-3}$ \\
Hydrogen concentration & $N_{v 0}$ & $2 \times 10^{21} \mathrm{~cm}^{-3} \mathrm{eV}^{-1}$ \\
Density of states at VB edge & $E_{v 0}$ & $56 \mathrm{meV}^{21}$ \\
Valence band tail slope & $N_{c 0}$ & $2 \times 10^{21} \mathrm{~cm}^{-3} \mathrm{eV}^{-1}$ \\
Density of states at CB edge & $E_{c 0}$ & $30 \mathrm{meV}^{-1}$ \\
Conductance band tail slope & $E_{0}$ & $55 \mathrm{meV}^{6}$ \\
Mayer-Neldel energy & $e_{00}$ & $2 \times 10^{6} \mathrm{~s}^{-1}$ \\
Exponential prefactor & $c_{n}^{+}$ & $5.5 \times 10^{-8} \mathrm{~cm}^{3} \mathrm{~s}^{-1}$ \\
Capture rates & $c_{p}^{0}$ & $1.1 \times 10^{-9} \mathrm{~cm}^{3} \mathrm{~s}^{-1}$ \\
& & \\
\hline \hline
\end{tabular}

The nonequilibrium occupancy function $f^{0 *}$

$$
f^{0 *}(E, n, p)=\frac{1}{1+\frac{e_{n}^{0}+p c_{p}^{0}}{e_{p}^{+}+n c_{n}^{+}}+\frac{e_{p}^{0}+n c_{n}^{0}}{e_{n}^{-}+p c_{p}^{-}}}
$$

also replaces $f^{0}$ in the integral (12) but the scaling factor $\gamma_{S}^{*}$ is essentially the same as $\gamma_{S}$. The symbols $c_{n}^{0}, c_{p}^{0}, c_{n}^{+}$, and $c_{p}^{-}$are the capture coefficients of electrons and holes into charged and neutral traps, and the associated emission rates are $e_{n}^{0}, e_{p}^{0}, e_{n}^{-}$, and $e_{p}^{+}$. The deviation from the equilibrium state is determined by the product of nonequilbrium concentrations of electrons $(n)$ and holes $(p), \quad[(n p) /$ $\left.\left(n_{0} p_{0}\right)\right]^{\rho, k T / 2 E_{v 0}}$. This product is the driving force for the generation of metastable defects.

Using formula (17) and the parameters obtained as the best fit for annealed state, we simulated two charge DLTS spectra after $20 \mathrm{~h} \mathrm{LS}$ spectra are saturated and a new steady-

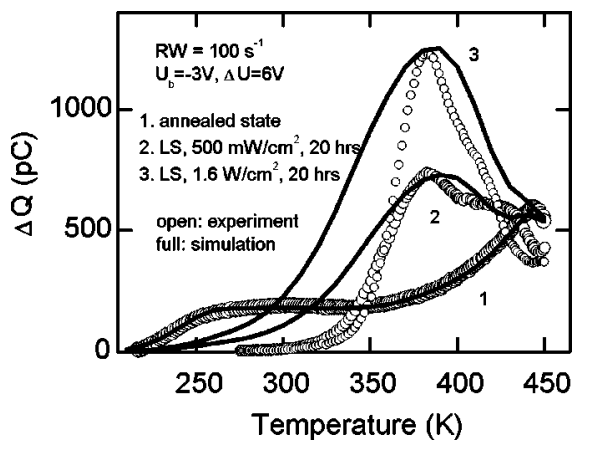

FIG. 4. Simulated charge DLTS spectra (full line) obtained as the best fit to experimental data points on the basis of the improved defect-pool model for $a$-Si:H by Powell and Dean (Ref. 7) and Schumm (Ref. 9). A annealed state and LS: light soaked state only by Schumm (Ref. 9) model (curves 6 and 7 in Fig. 1). Note that the spectrum for the annealed state is identical for both models but for different values of fitting parameters (see Table II).

state distribution $D(E)$ is reached. For these LS states other four parameters were fitted, namely, the capture rates $c_{n}^{0}, c_{p}^{-}$ and the quasi-Fermi levels $E_{F n}, E_{F p}$ arisen due to illumination. The reason for including those capture rates in fitted parameters is their substantial impact on the gap state distribution. In contrary, capture rates $c_{n}^{+}$and $c_{p}^{0}$ practically do not effect on this distribution. The position of the quasi Fermi levels in the gap is mainly determined by the light intensity and concentration of the gap states. It should be noted that this simulation was performed at temperature of $300 \mathrm{~K}$.

A set of experimental and theoretical charge DLTS spectra is shown in Fig. 4. Figure 5 shows the electronic density of states $g(E)$ of the annealed and two light-soaked states of undoped $a-\mathrm{Si}: \mathrm{H}$, calculated on the basis of defect-pool model parameters corresponding to the best fit of the experimental charge DLTS spectra in the simulation process. Inspection of Figs. 4 and 5, and obtained values of fitted parameters lead to several conclusions. Powell and Dean's and Schumm's models ${ }^{7,9}$ can give identical gap state distribution though small differences in the fitted parameters are required (see Table II). Our simulations confirm the excess of charged $D_{h}$

TABLE II. The parameters pool position $E_{p}$, pool width $\sigma$, correlation energy $U$, and amplitude constant $\Delta Q_{0}$ were obtained as the best fit to the experimental spectra. Note that four other fitting parameters, capture rates $c_{n}^{0}, c_{p}^{-}$and quasi-Fermi levels $E_{F n}, E_{F p}$ were used for the simulation of the charge DLTS spectra after LS by Schumm's model. ${ }^{9}$ Two values of the quasi-Fermi levels were obtained as the best fit to experimental spectra 6 and 7 in Fig. 1.

\begin{tabular}{lccc}
\hline \hline & & \multicolumn{2}{c}{ Value } \\
Fitted parameter & Symbol & Powell\&Dean'93 & Schumm'94 \\
\hline Pool position & $E_{p}$ & $1.39 \mathrm{eV}$ & $1.395 \mathrm{eV}$ \\
Pool width & $\sigma$ & $0.186 \mathrm{eV}$ & $0.202 \mathrm{eV}$ \\
Correlation energy & $U$ & $0.146 \mathrm{eV}$ & $0.170 \mathrm{eV}$ \\
Amplitude constant & $\Delta Q_{0}$ & $1.5 \times 10^{-25} \mathrm{C} \mathrm{cm}^{3}$ & $9.0 \times 10^{-26} \mathrm{C} \mathrm{cm}^{3}$ \\
Capture rates & $c_{n}^{0}$ & & $5 \times 10^{-10} \mathrm{~cm}^{3} \mathrm{~s}^{-1}$ \\
& $c_{p}^{-}$ & & $5 \times 10^{-8} \mathrm{~cm}^{3} \mathrm{~s}^{-1}$ \\
Quasi-Fermi level for electrons & $E_{F n}$ & & $1.335 \mathrm{eV}^{\prime} 1.370 \mathrm{eV}$ \\
Quasi-Fermi level for holes & $E_{F p}$ & & $0.86 \mathrm{eV} / 0.81 \mathrm{eV}$ \\
\hline \hline
\end{tabular}




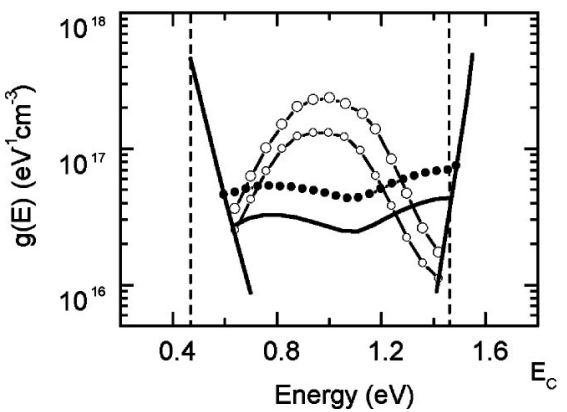

FIG. 5. Electronic density of states $g(E)$, of the annealed [(Powell and Dean's model (Ref. 7), 0 - Schumm's model (Ref. 9)] and light soaked states [○○○ Schumm's model (Ref. 9)] of undoped a-Si:H, calculated on the basis of defect-pool model parameters which correspond to the best fit to the experimental DLTS spectra. Vertical dashed lines indicate the energy limits for calculation of the charge DLTS specta.

and $D_{e}$ defects over the neutral $D_{z}$ ones, predicted by improved defect-pool model, ${ }^{7,9}$. For the annealed state lower total density of states is obtained by Powell and Dean ${ }^{7}$ in comparison with Schumm ${ }^{9}$ (by factor of 1.6). For the LS state Schumm's model overestimates the density of charged (mainly $D_{h}$ ) gap states. In addition to the position of the quasi-Fermi levels, the distribution of gap states is sensitive mainly to the capture rates of the second electron $c_{n}^{0}$ and the first hole $c_{p}^{-}$.

\section{CONCLUSIONS}

On the basis of facts mentioned above it is possible to conclude that the results of charge DLTS and ESR techniques correlate well. Light soaking time dependence of the intensity of the charge DLTS and ESR signals can be explained by a downward shift of the Fermi level towards midgap in the early stage of the light soaking due to the removal of $D_{h}$ defects. Corresponding defect states play the role of donors and their decay is reason for well-known decrease of dark and photoconductivities in SWE. Our preliminary simulation with the ASA software package ${ }^{26}$ shows that changes of $g(E)$ with the accompanying downward shift of the Fermi level presented in this paper quite naturally explains of the recombination kinetics change reported in recent works. ${ }^{15-18}$ The authors try to explain this changes by creation of new charged defects. Since they assume their existence indirectly from measurements of optical absorption and electron mobility-lifetime product, their interpretation is less probable.

Simulations of the experimental charge DLTS spectra using different modifications of the defect-pool model ${ }^{7,9}$ give a very good fit to spectra obtained on undoped $a$-Si:H in the annealed state whilst there is some discrepancy between the experimental and simulated spectra for the light-soaked state. Apparently, the defect-pool model overestimates the presence of charged defects $\left(D_{h}, D_{e}\right)$ in the light-soaked state. Therefore, the presented comparison of defect pool model with charge DLTS offers a valuable stimulus for theoretical description of paradoxical decay of $D_{h}$ states in the early stage of SWE, which is probably the key to the explanation of this effect.

\section{ACKNOWLEDGMENTS}

One of the authors (V.N.) acknowledges the support of the Asahi Shimbun Foundation in carrying out this work. This work was partly supported by the VEGA grant agency of Slovak Republic under Project Nos. 2/1013/21 and 2/1119/ 21.
${ }^{1}$ D.V. Lang, J.D. Cohen, and J.P. Harbison, Phys. Rev. B 25, 5285 (1982).

${ }^{2}$ J.D. Cohen and D.V. Lang, Phys. Rev. B 25, 5321 (1982).

${ }^{3}$ J.D. Cohen, J.P. Harbison, and K.W. Wecht, Phys. Rev. Lett. 48, 109 (1982).

${ }^{4}$ D.V. Lang, J.D. Cohen, and J.P. Harbison, Phys. Rev. Lett. 48, 421 (1982).

${ }^{5}$ T. Kamei, N. Hata, A. Matsuda, T. Uchiyama, S. Amano, K. Tsukamoto, Y. Yoshita, and T. Hirao, Appl. Phys. Lett. 68, 2380 (1996).

${ }^{6}$ V. Nádaždy, R. Durný, and E. Pinčík, Phys. Rev. Lett. 78, 1102 (1997).

${ }^{7}$ M.J. Powell and S.C. Deane, Phys. Rev. B 48, 10815 (1993).

${ }^{8}$ J.M. Asensi and J. Andreu, Phys. Rev. B 47, 13295 (1993).

${ }^{9}$ G. Schumm, Phys. Rev. B 49, 2427 (1994).

${ }^{10}$ M.J. Powell and S.C. Deane, Phys. Rev. B 53, 10121 (1996).

${ }^{11}$ K. Winer, Phys. Rev. B 41, 12150 (1990).

${ }^{12}$ V. Nádaždy, R. Durný, I. Thurzo, and Pinčık, J. Non-Cryst. Solids 227-230, 316 (1998).

${ }^{13}$ K. Shimizu, T. Tabuchi, M. Iida, and H. Okamoto, J. Non-Cryst. Solids 227-230, 267 (1998).
${ }^{14}$ N. Hata, T. Kamei, H. Okamoto, and A. Matsuda, in Amorphous and Microcrystalline Silicon Technology-1997, edited by S. Wagner et al., Mater. Res. Soc. Symp. Proc. No. 467 (Materials Research Society, Warrendale, 1997), p. 61.

${ }^{15}$ S. Heck and H.M. Branz, Appl. Phys. Lett. 79, 3080 (2001).

${ }^{16} \mathrm{~S}$. Heck and H.M. Branz, in Amorphous and Heterogeneous Silicon - Based Films-2001, edited by M. Stutzmann et al., Mater. Res. Soc. Symp. Proc. No. 664 (Materials Research Society, Warrendale, 2001).

${ }^{17}$ J. Pearce, X. Niu, R. Koval, G. Ganguly, D. Carlson, R.W. Collins, and C.R. Wronski, in Amorphous and Heterogeneous Silicon - Based Films-2001, edited by M. Stutzmann et al. Mater. Res. Soc. Symp. Proc. No. 664 (Materials Research Society, Warrendale, 2001).

${ }^{18}$ R. Koval, X. Niu, J. Pearce, L. Jiao, G. Ganguly, J. Yang, S. Guha, R.W. Collins, and C.R. Wronski, in Amorphous and Heterogeneous Silicon Thin Films, edited by R. W. Collins et al., Mater. Res. Soc. Symp. Proc. No. 609 (Materials Research Society, Warrendale, 2000), p. A15.5.

${ }^{19}$ R. Durný, E. Pinčík, V. Nádaždy, M. Jergel, J. Shimizu, M. Kumeda, and T. Shimizu, Appl. Phys. Lett. 77, 1783 (2000). 
${ }^{20}$ I. Thurzo and M. Grendel, Meas. Sci. Technol. 3, 726 (1992).

${ }^{21}$ V. Nádaždy and I. Thurzo, Phys. Status Solidi A 127, 167 (1991).

${ }^{22}$ J-H. Zhou, M. Kumeda, and T. Shimizu, Phys. Rev. B 53, 7267 (1996).

${ }^{23}$ L. Jiao, H. Liu, S. Semoushikina, Y. Lee, and C.R. Wronski, Appl. Phys. Lett. 69, 3713 (1996).

${ }^{24}$ H. Fritzsche, Solid State Commun. 94, 953 (1995).

${ }^{25}$ V. Nádaždy, R. Durný, E. Pinčík, I. Thurzo, M. Kumeda, and T.
Shimizu, J. Non-Cryst. Solids 266-269, 558 (2000).

${ }^{26}$ M. Zeman, J.A. Willemen, L.L.A. Vosteen, G. Tao, and J.W. Metselaar, Solar Energy Materials and Solar Cells 46, 81 (1997).

${ }^{27}$ D. V. Lang, in Topics in Applied Physics, edited by P. Braunlich (Springer, Berlin, 1979), Vol. 37, p. 93.

${ }^{28}$ A. Yelon, B. Movaghar, and H.M. Branz, Phys. Rev. B 46, 12244 (1992). 